\title{
Clusters of Cesium-Lead-lodide Perovskites in the Zeolite Matrix
}

\author{
Igor Dmitruk,* Yelyzaveta Vikhrova, Andriy Dmytruk, Nataliya Berezovska, Yuri Barnakov, Polina Vartik, \\ and Rodion V. Belosludov
}

Cite This: ACS Omega 2021, 6, 27711-27715

Read Online

ABSTRACT: Small clusters of cesium-lead-iodide perovskites (CLIPs) have been prepared in the $\mathrm{Na}-\mathrm{X}$ zeolite matrix and studied by means of spectroscopy and quantum chemical modeling. Regularity of pores in single crystals of zeolite assures the formation of clusters of a certain size. By the first-principles quantum chemical calculations, we have determined that clusters $\mathrm{Cs}_{4} \mathrm{PbI}_{6}, \mathrm{Cs}_{5} \mathrm{~Pb}_{2} \mathrm{I}_{9}$, and probably $\mathrm{Cs}_{7} \mathrm{~Pb}_{5} \mathrm{I}_{16}$ with a size of $0.74,1.29$, and $1.36 \mathrm{~nm}$, respectively, have elevated stability compared to other species, and they fit into the pores of $\mathrm{Na}-\mathrm{X}$ zeolite. Electronic energy spectra of these clusters have been calculated and compared with experimentally measured ones.

\section{INTRODUCTION}

Perovskite materials of different dimensionalities are promising due to their useful electronic transitions and insensitivity of their electronic energy spectrum to structure defects. ${ }^{1-3}$ However, they require protection from the environment. This can be accomplished by surfactant coverage or by incorporation into a porous matrix, for instance, mesoporous silica matrix. ${ }^{4}$ Pores of the small size allow the preparation of nanoparticles of perovskites that open up an additional possibility of controlling their energy spectrum via quantum size effect. Although nanoparticles of perovskites have been studied in a number of papers (see, e.g., refs 2, 3, 5-15, and 20 ), nevertheless, the smallest possible nanoclusters consisting of up to 20 atoms are still rather unexplored. As an example of $0 D$ perovskite theoretical calculations, we can mention refs 25 and 26. Several papers report incorporation of perovskite nanoparticles into zeolite. ${ }^{21,22,24,27}$

We attempt to impregnate zeolite single crystals with CLIP. The small size of pores in zeolite supports incorporation of OD perovskite clusters consisting of one-two tens of atoms, such as $\mathrm{Cs}_{4} \mathrm{PbI}_{6}, \mathrm{Cs}_{5} \mathrm{~Pb}_{2} \mathrm{I}_{9}$, and $\mathrm{Cs}_{7} \mathrm{~Pb}_{5} \mathrm{I}_{16}$. Quantum chemical calculations were performed to optimize the geometry and calculate other properties of $0 \mathrm{D}$ perovskites. The calculated absorption and luminescence spectra are compared with the measured ones on the synthesized CLIP@zeolite nanocomposites.

Zeolites provide the matrix to obtain extremely small clusters in the pores with a diameter up to $15 \AA$. Zeolites are crystalline aluminosilicates with channels and cavities that can vary in the range from 3 to $15 \AA .{ }^{16}$ Cavity size depends on the type of aluminosilicate framework, $\mathrm{Si} / \mathrm{Al}$ ratio, origin of ion-exchange cations, and so forth. $\mathrm{Na}-\mathrm{X}$ zeolite, which has been used in the present paper, has a $\mathrm{Si} / \mathrm{Al}$ ratio equal to 1 , $F d 3 m$ symmetry, and two types of cages: one is a sodalite cage,

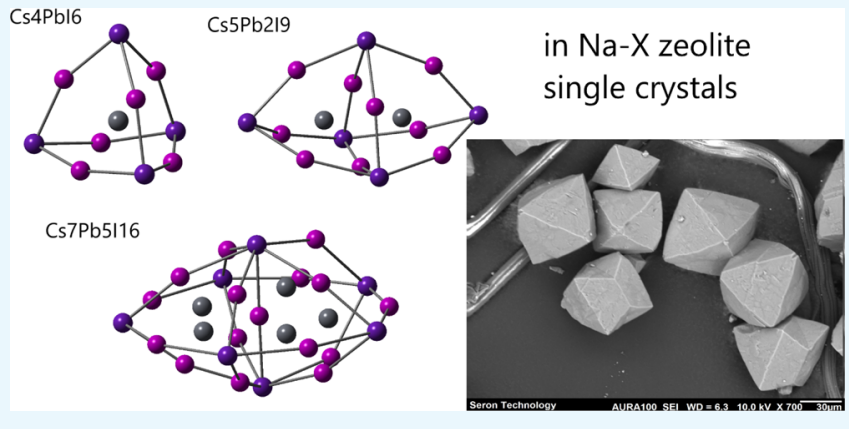

truncated octahedron with a diameter of $8 \AA$, and a supercage, which is formed by the connection of sodalites in a diamondlike structure with a diameter of about $13 \AA \AA^{17}$ All cages are interconnected by shared small windows and arranged regularly. Thus, the cages can be used for the fabrication of small nanoclusters. Even the smallest perovskite nanoclusters $\left(\mathrm{Cs}_{4} \mathrm{PbI}_{6}\right)$ barely fit into the small $(8 \AA)$ sodalite cages. Therefore, it is natural to assume that mostly, the supercages can be the hosts for the CLIP nanoclusters.

\section{RESULTS AND DISCUSSION}

2.1. Sample Preparation and Measurements. To date, as far as we know, there are a few examples of the synthesis of perovskites in zeolites reported. ${ }^{21,22}$ They have performed twostep synthesis from solution. In the present report, we used a simpler one-step technique very similar to that used in the synthesis of perovskites in mesoporous glass. ${ }^{4} \mathrm{CsI}$ and $\mathrm{PbI}_{2}$ have been dissolved in dimethylformamide (DMF) to form 0.2 $\mathrm{M}$ stock solution of CLIP $\mathrm{CsPbI}_{3}$. Zeolite crystals were immersed in CLIP solution and left for a certain amount of time. It should be emphasized that immersion time must be long enough (about 12-24 h) because of small diameter of channels between cavities in zeolite crystals and slow diffusion of ions through channels. It happens because ions can be adsorbed on the walls of channels, and it takes time for

Received: June 1, 2021

Accepted: October 5, 2021

Published: October 12, 2021 
desorption-diffusion-adsorption processes repeated many times. Temperature of the sample during percolation is also important. From one side, increase of temperature speeds up desorption and diffusion processes, but from the other side, it decreases the solubility of the perovskite. It opens up possibility to control penetration of perovskites into zeolites by changing temperature in the next studies. In the present report, we used room temperature. Then, the excess solution was removed using filter paper. The samples were heat-treated at $150{ }^{\circ} \mathrm{C}$ for complete drying.

Initial characterization of zeolite samples was performed with a scanning electron microscope AURA-100 (Seron Technology) and a transmission electron microscope JEOL 100CX II. Comparison of images of zeolite crystals before and after treatment in CLIP solution does not reveal any changes. Thus, we can conclude that the crystalline structure of zeolite is preserved. It is also confirmed by electron diffraction (ED) (Figure S3). Unfortunately, we have no technology for preparation of the slices of zeolite crystals thin enough for high-resolution transmission electron microscopy (TEM) observation of CLIP clusters in the pores.

Photoluminescence (PL) spectra of zeolite single crystals with CLIP have been measured with aspectrometer based on MDR-3 monochromator under $405 \mathrm{~nm} \mathrm{cw}$ semiconductor laser and $337 \mathrm{~nm}$ pulsed nitrogen laser excitation at room temperature. The control PL spectra of zeolite without perovskites have been measured (Figure S6). They gave negligible intensity in all studied spectral ranges. Luminescence excitation spectra have been measured with a Shimadzu RF6000 spectrofluorophotometer.

2.2. Quantum Chemistry Calculations. Correct modeling of the perovskite nanoparticles is an important part of the study to explain the optical and electronic processes in the nanoparticles. First of all, we have to say that the effective mass approximation, ${ }^{18}$ which is often used to describe an exciton quantum confinement, analogous to a particle in a "box", gives substantial deviation from the experiment for small particles, so it requires a more accurate approach. Going beyond the abovementioned theory, it is better to analyze the charge redistribution occurring in these excitations for a more accurate description of the optical properties of nanoparticles.

Today, many groups are conducting quantum chemical calculations to correctly describe $0 \mathrm{D}, 1 \mathrm{D}$, and $2 \mathrm{D}$ perovskites and find the optimal basis that will be suitable for perovskites with any chemical composition. However, reaching agreement with experimental electronic spectra is still an actual task. Extensive modeling of the smallest $0 \mathrm{D}$ perovskite cluster led us to conclusion that it has the structure of appearance $\mathrm{A}_{4} \mathrm{BX}_{6}$. Its composition is similar to $\mathrm{Cs}_{4} \mathrm{PbX}_{6}$ nanoparticles ${ }^{23}$ which behave like an insulator. However, we have to emphasize the difference in energy spectra and optical properties of $\mathrm{Cs}_{4} \mathrm{PbX}_{6}$ nanoparticles ${ }^{23}$ and extremely small clusters $\mathrm{Cs}_{4} \mathrm{PbX}_{6}$ consisting of 11 atoms.

The most stable structure of the $\mathrm{A}_{4} \mathrm{BX}_{6}$ cluster is the tetrahedron with metal $\mathrm{B}$ at the center, alkali $\mathrm{A}$ at the vertices, and halogens $\mathrm{X}$ at the edges. The structure of larger perovskite clusters consists of a certain number of elementary building blocks mentioned above. Therefore, to simplify the construction of larger clusters, it was necessary to optimize this elementary cell and to create larger clusters from these. The structural and spectral characteristics were calculated using the Gaussian 16 program. ${ }^{19}$ First of all, the geometry of the nanoclusters was optimized. The structure and energy optimization were performed using density functional theory (DFT). A generalized gradient approximation in the PerdueBurke-Ernzerhof (PBE) functional form ${ }^{19}$ with the scalar relativistic effective Stuttgart/Dresden base potential (SDD) is chosen.

According to the pseudopotential theory used in the DFT method, a strong ionic potential can be replaced by a much weaker potential, a pseudopotential describing all the pronounced properties of the motion of valence electrons in the solid state. The potential is introduced in such a way that the multielectron problem is replaced by the self-consistent field problem, and all electronic states are subdivided into the framework and valence ones. The core electrons are excluded from consideration of the assumption that their charge density does not respond to changes in the chemical environment. However, this approximation is very rough, so we use a generalized gradient approximation to account for the heterogeneity of the real electron density.

The optimized structures of small perovskite clusters $\mathrm{Cs}_{4} \mathrm{PbI}_{6}, \mathrm{Cs}_{5} \mathrm{~Pb}_{2} \mathrm{I}_{9}$, and $\mathrm{Cs}_{7} \mathrm{~Pb}_{5} \mathrm{I}_{16}$ chosen from many tens of modeled clusters as ones with the largest binding energies are presented in Figures 1, 2 and their calculated absorption spectra are presented in Figure 3. These clusters are small enough to fit into supercages of zeolite (Figure 2).
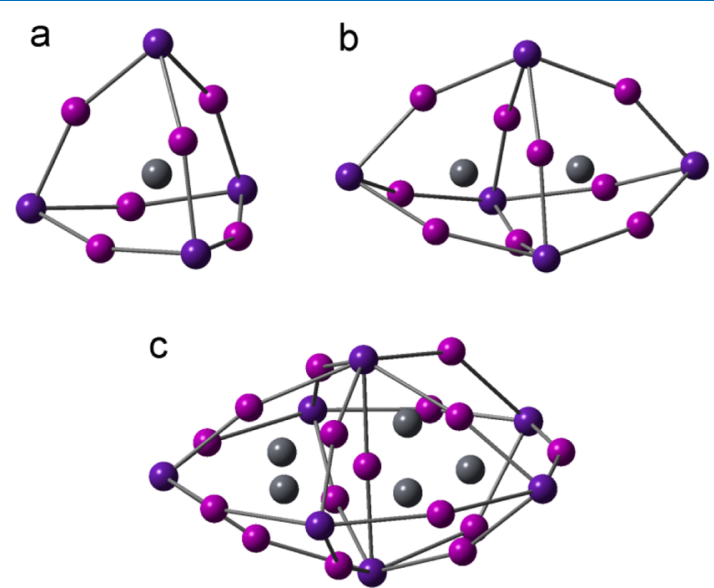

Figure 1. Optimized structures of small perovskite clusters $\mathrm{Cs}_{4} \mathrm{PbI}_{6}$ (a), $\mathrm{Cs}_{5} \mathrm{~Pb}_{2} \mathrm{I}_{9}$ (b), and $\mathrm{Cs}_{7} \mathrm{~Pb}_{5} \mathrm{I}_{16}$ (c).

\subsection{Luminescence of Perovskite Clusters in Zeolites.} An attempt to synthesize perovskite nanoparticles has met all expectations. Unlike porous silica, in zeolites, the pores are uniform and have a definite direction, a strict arrangement, and

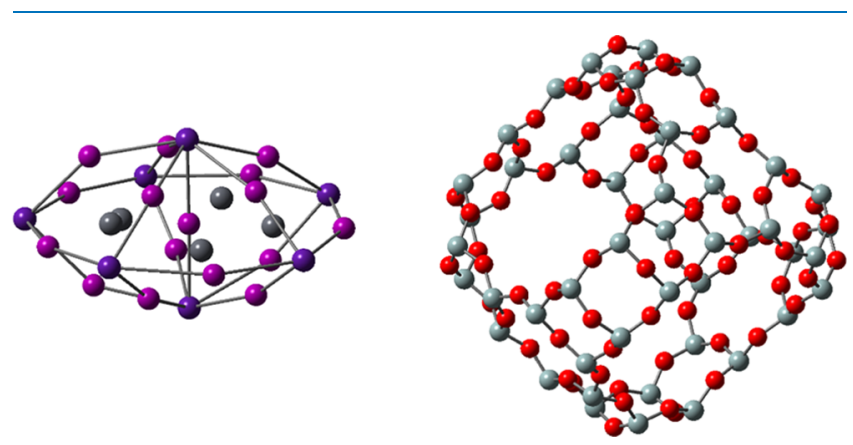

Figure 2. Perovskite cluster $\mathrm{Cs}_{7} \mathrm{~Pb}_{5} \mathrm{I}_{16}$ and the inner $\mathrm{Si}-\mathrm{O}$ shell of the $\mathrm{Na}-\mathrm{X}$ zeolite supercage. 


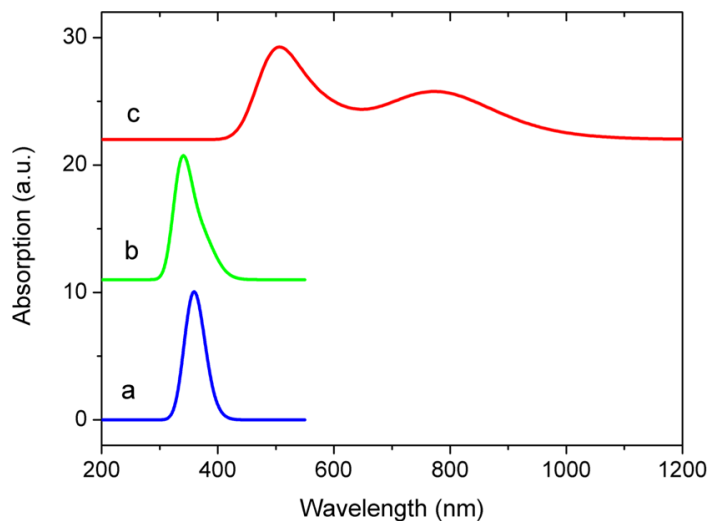

Figure 3. Calculated absorption spectra of small perovskite clusters: $\mathrm{Cs}_{4} \mathrm{PbI}_{6}(\mathrm{a}), \mathrm{Cs}_{5} \mathrm{~Pb}_{2} \mathrm{I}_{9}(\mathrm{~b})$, and $\mathrm{Cs}_{7} \mathrm{~Pb}_{5} \mathrm{I}_{16}$ (c).

very small dimensions. For zeolite crystals treated in perovskite solution of a concentration of $0.2 \mathrm{M}$, there is one narrow luminescence band at $425 \mathrm{~nm}$ (Figure 4). It should be

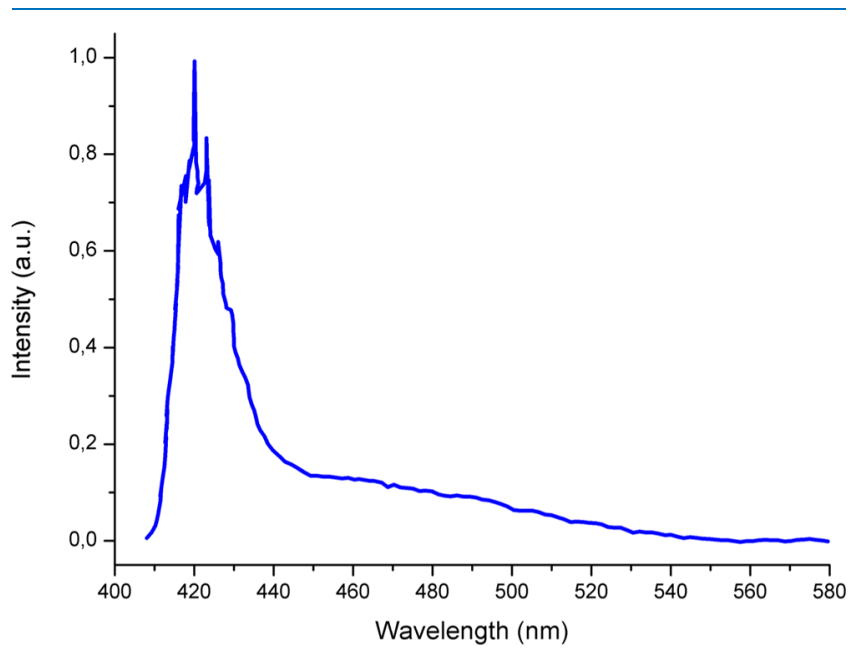

Figure 4. Luminescence of $\mathrm{Na}-\mathrm{X}$ zeolite crystals impregnated with CLIP under $405 \mathrm{~nm} \mathrm{cw}$ laser excitation.

emphasized that the $\mathrm{Na}-\mathrm{X}$ zeolite is a wide band gap crystal and it does not absorb $405 \mathrm{~nm}$ emission of semiconductor laser and does not provide any noticeable luminescence (Figure S6). Only zeolite with perovskites provides luminescence presented in Figure 4. The shift of this band into the short wavelength region compared to bulk CLIP indicates a decrease in the size of the clusters. It is manifestation of quantum size effect.

A narrow band at $425 \mathrm{~nm}$ may be interpreted as luminescence of perovskite clusters (most likely $\mathrm{Cs}_{4} \mathrm{PbI}_{6}$ ) in the pores, while a longer-wavelength shoulder can be attributed to another kind of clusters (probably $\mathrm{Cs}_{5} \mathrm{~Pb}_{2} \mathrm{I}_{9}$ ) or to the perovskite film and larger nanocrystals at the surface of zeolite crystals (Figure S2). We have noticed that the PL spectrum depends on the excitation source. In Figure 5, luminescence under pulsed nitrogen UV laser $(337 \mathrm{~nm})$ is presented. Approximately, in the same spectral range, two blue bands are observed at 470 and $510 \mathrm{~nm}$, respectively. Probably, such a sensitivity of the PL spectrum to excitation wavelength is caused by the difference of absorption spectra of different perovskite clusters and nanoparticles.

We have performed DFT calculations of $\mathrm{Cs}_{4} \mathrm{PbI}_{6}, \mathrm{Cs}_{5} \mathrm{~Pb}_{2} \mathrm{I}_{9}$, and $\mathrm{Cs}_{7} \mathrm{~Pb}_{5} \mathrm{I}_{16}$ OD perovskite clusters to compare their

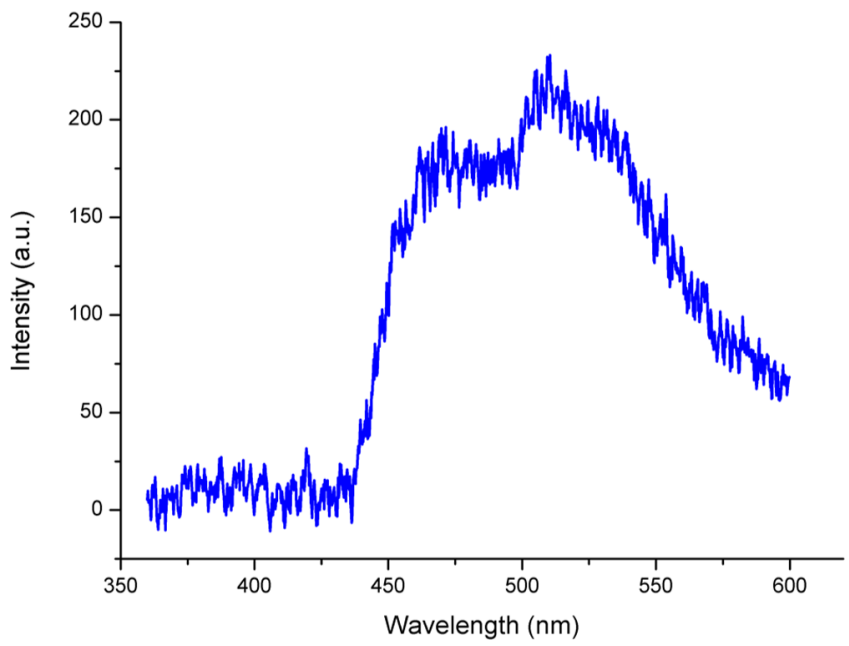

Figure 5. Luminescence of $\mathrm{Na}-\mathrm{X}$ zeolite crystals impregnated with CLIP under $337 \mathrm{~nm} \mathrm{~N}_{2}$ laser excitation.

predicted energy spectra with the experimental results presented above. Comparison of calculated (Figure 3) and experimental (Figure 4) spectra suggests that the most probable composition of perovskite clusters in the pores of zeolite is $\mathrm{Cs}_{4} \mathrm{PbI}_{6}$ and $\mathrm{Cs}_{5} \mathrm{~Pb}_{2} \mathrm{I}_{9}$. A larger cluster $\mathrm{Cs}_{7} \mathrm{~Pb}_{5} \mathrm{I}_{16}$ has the electronic energy spectrum shifted considerably to the lowenergy side and consisting of two rather broad bands. It is interesting that absorption spectra of $\mathrm{Cs}_{4} \mathrm{PbI}_{6}$ and $\mathrm{Cs}_{5} \mathrm{~Pb}_{2} \mathrm{I}_{9}$ clusters have very close position of the main absorption peak centered near $350 \mathrm{~nm}$. However, cluster $\mathrm{Cs}_{5} \mathrm{~Pb}_{2} \mathrm{I}_{9}$ (Figure $3 \mathrm{~b}$ ) has an asymmetric peak with a long-wavelength shoulder that resembles better the experimental one (Figure 4).

We cannot perform direct measurement of the absorption spectrum of the zeolite-CLIP sample because it consists of small crystals (Figure S1, S2) with a high refractive index that causes strong light scattering. However, we can measure the luminescence excitation spectrum instead. Obtained results are presented in Figure 6. Excitation spectra contain two bands at 320 and $360 \mathrm{~nm}$ and a short-wavelength shoulder. It is easy to notice that the ratio of intensities of two bands and their shapes change, while monitoring wavelength is changed (469

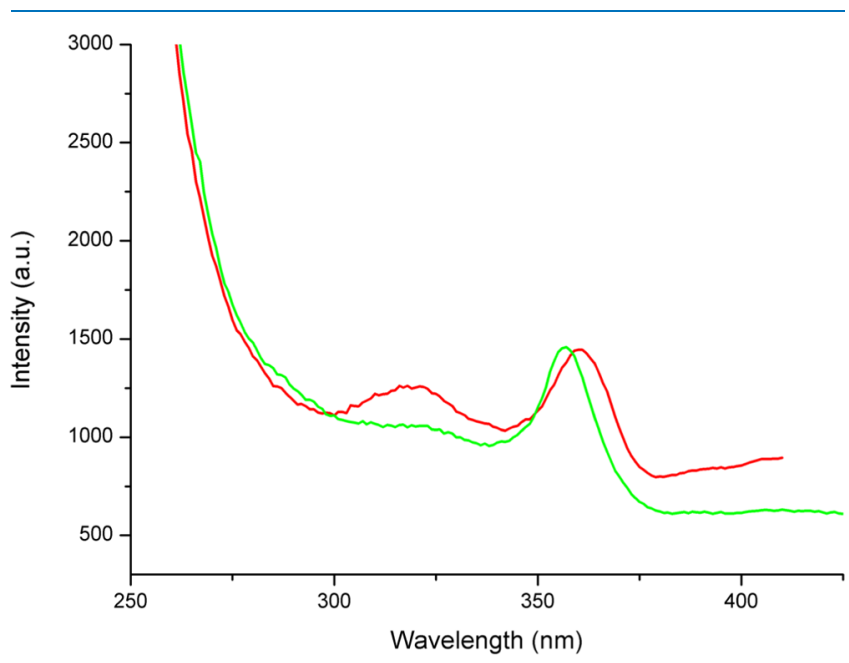

Figure 6. Excitation spectra of luminescence of $\mathrm{Na}-\mathrm{X}$ zeolite crystals impregnated with CLIP at $469 \mathrm{~nm}$ (red curve) and $500 \mathrm{~nm}$ (green curve). 
and $500 \mathrm{~nm}$, respectively). The observed structure of PL and excitation spectra that contain two bands is in agreement with the results of theoretical calculations that predict the existence of two most stable CLIP clusters $\mathrm{Cs}_{4} \mathrm{PbI}_{6}$ and $\mathrm{Cs}_{5} \mathrm{~Pb}_{2} \mathrm{I}_{9}$ that fit into zeolite cages. A short-wavelength shoulder in excitation spectra can be caused by transitions into the higher excited states that also appear in the results of DFT calculations (Figure S5).

Another possibility to compare experimental and calculation results on clusters' spectra is to estimate Stokes shift between absorption and emission transitions. It is possible to estimate expected Stokes shift by means of quantum chemical calculations-an optimization of cluster in the excited electronic state. However, such calculations are much more time-consuming and provide less accuracy. Our preliminary results demonstrate a Stokes shift of about $1.13 \mathrm{eV}$ (that is about 2 times larger than experimentally observed one) for $\mathrm{Cs}_{4} \mathrm{PbI}_{6}$ and much larger values for $\mathrm{Cs}_{5} \mathrm{~Pb}_{2} \mathrm{I}_{9}$ and $\mathrm{Cs}_{7} \mathrm{~Pb}_{5} \mathrm{I}_{16}$, which can reflect large deformation of electronic bands and possibility of nonradiative de-excitation in these clusters (Figure S4). The difference of calculated and experimentally observed Stokes shift shows the need for more accurate calculations.

There are sharp lines on the $425 \mathrm{~nm}$ band as well (Figure 4). Their origin is not clear yet. They can correspond to collective emission (like in ref 14) of many regularly arranged nanoclusters from the areas of zeolite crystals with high filling of cages with perovskite. Figure S2).

\section{CONCLUSIONS}

Small perovskite clusters have been grown in the matrix of $\mathrm{Na}-\mathrm{X}$ zeolite single crystals. Luminescence of these clusters demonstrates blue bands at 425-500 $\mathrm{nm}$ with sharp lines and a long-wavelength shoulder attributed to the perovskite film on the surface. Quantum chemical calculations have been performed to determine the size and energy spectrum of the smallest possible perovskite clusters that can fit into supercages of zeolite and to compare with experimental results. From the results of these calculations, we conclude that the largest binding energies per atom demonstrate tetrahedral clusters $\mathrm{Cs}_{4} \mathrm{PbI}_{6}$ and larger ones built from 2 or 5 tetrahedrons, namely, $\mathrm{Cs}_{5} \mathrm{~Pb}_{2} \mathrm{I}_{9}$ and $\mathrm{Cs}_{7} \mathrm{~Pb}_{5} \mathrm{I}_{16}$. Calculations of the electronic excited states and comparison with experimental luminescence and excitation spectra suggest that two smaller clusters $\mathrm{Cs}_{4} \mathrm{PbI}_{6}$ and $\mathrm{Cs}_{5} \mathrm{~Pb}_{2} \mathrm{I}_{9}$ can appear in our measurements.

\section{ASSOCIATED CONTENT}

\section{SI Supporting Information}

The Supporting Information is available free of charge at https://pubs.acs.org/doi/10.1021/acsomega.1c02864.

Additional figures, spectra, and tables with binding energies and coordinates of atoms of the optimized structure of perovskite nanoclusters (PDF)

\section{AUTHOR INFORMATION}

\section{Corresponding Author}

Igor Dmitruk - Faculty of Physics, National Taras Shevchenko University of Kyiv, Kyiv 01601, Ukraine; (1) orcid.org/0000-0001-9482-8746;

Email: igor_dmitruk@univ.kiev.ua

\section{Authors}

Yelyzaveta Vikhrova - Faculty of Physics, National Taras Shevchenko University of Kyiv, Kyiv 01601, Ukraine

Andriy Dmytruk - Institute of Physics, National Academy of Sciences of Ukraine, Kyiv 03028, Ukraine

Nataliya Berezovska - Faculty of Physics, National Taras Shevchenko University of Kyiv, Kyiv 01601, Ukraine

Yuri Barnakov - SETC, IPG Photonics, Birmingham, Alabama 35211, United States

Polina Vartik - Faculty of Physics, National Taras Shevchenko University of Kyiv, Kyiv 01601, Ukraine

Rodion V. Belosludov - Institute for Materials Research, Tohoku University, Sendai, Miyagi 980-8577, Japan; (1) orcid.org/0000-0003-4661-9194

Complete contact information is available at:

https://pubs.acs.org/10.1021/acsomega.1c02864

\section{Author Contributions}

The manuscript was written through contributions of all authors. All authors have given approval to the final version of the manuscript.

\section{Notes}

The authors declare no competing financial interest.

\section{ACKNOWLEDGMENTS}

I.D. and R.V.B. are grateful to the crew of Center for Computational Materials Science, Cooperative Research and Development Center for Advanced Materials and E-IMR center at the Institute for Materials Research, Tohoku University, for continuous support. I.D. and N.B. acknowledge funding from the Ministry of Education and Science of Ukraine (Project State reg. no. 0119U100300). Authors are thankful to Dr. A. Kotko for TEM and ED measurements and Prof. O. Yeshchenko for excitation spectroscopy measurements.

\section{REFERENCES}

(1) Kovalenko, M. V.; Protesescu, L.; Bodnarchuk, M. I. Properties and potential optoelectronic applications of lead halide perovskite nanocrystals. Science 2017, 358, 745-750.

(2) Akkerman, Q. A.; Abdelhady, A. L.; Manna, L. ZeroDimensional Cesium Lead Halides: History, Properties, and Challenges. J. Phys. Chem. Lett. 2018, 9, 2326-2337.

(3) Iso, Y.; Isobe, T. Review-Synthesis, Luminescent Properties, and Stabilities of Cesium Lead Halide Perovskite Nanocrystals. ECS J. Solid State Sci. Technol. 2018, 7, R3040-R3045.

(4) Dirin, D. N.; Protesescu, L.; Trummer, D.; et al. Harnessing Defect-Tolerance at the Nanoscale: Highly Luminescent Lead Halide Perovskite Nanocrystals in Mesoporous Silica Matrixes. Nano Lett. 2016, 16, 5866-5874.

(5) Kshirsagar, B.; Jaykhedkar, N.; Jain, K.; et al. Green CsSnX3 (X $=\mathrm{Cl}, \mathrm{Br}, \mathrm{I}$ )-Derived Quantum Dots for Photovoltaic Applications: First-Principles Investigations. J. Phys. Chem. C 2021, 125, 25922606.

(6) Li, R.; Yu, J.; Wang, S.; et al. Surface modification of all-inorganic halide perovskite nanorods by a microscale hydrophobic zeolite for stable and sensitive laser humidity sensing. Nanoscale 2020, 12, 13360-13367.

(7) Swarnkar, A.; Marshall, A. R.; Sanehira, E. M.; et al. Quantum dot-induced phase stabilization of - $\mathrm{CsPbI} 3$ perovskite for highefficiency photovoltaics. Science 2016, 354, 92-95.

(8) Saidaminov, M. I.; Almutlaq, J.; Sarmah, S.; et al. Pure Cs4PbBr6: Highly Luminescent Zero-Dimensional Perovskite Solids. ACS Energy Lett. 2016, 1, 840-845.

(9) Becker, M. A.; Vaxenburg, R.; Nedelcu, G.; et al. Bright triplet excitons in caesium lead halide perovskites. Nature 2018, 553, 189. 
(10) Chen, Q.; Wu, J.; Ou, X.; et al. All-inorganic perovskite nanocrystal scintillators. Nature 2018, 561, 88-93.

(11) Kulkarni, S. A.; Mhaisalkar, S. G.; Mathews, N.; Boix, P. P. Perovskite Nanoparticles: Synthesis, Properties, and Novel Applications in Photovoltaics and LEDs. Small Methods 2019, 3, 1800231.

(12) Lin, H.; Zhou, C.; Tian, Y.; et al. Low-Dimensional Organometal Halide Perovskites. ACS Energy Lett. 2018, 3, 54-62.

(13) Huang, H.; Bodnarchuk, M. I.; Kershaw, S. V.; et al. Lead Halide Perovskite Nanocrystals in the Research Spotlight: Stability and Defect Tolerance. ACS Energy Lett. 2017, 2, 2071-2083.

(14) Rainò, G.; Becker, M. A.; Bodnarchuk, M. I.; et al. Superfluorescence from lead halide perovskite quantum dot superlattices. Nature 2018, 563, 671-675.

(15) Tamarat, P.; Bodnarchuk, M. I.; Trebbia, J.-B.; et al. The ground exciton state of formamidinium lead bromide perovskite nanocrystals is a singlet dark state. Nat. Mater. 2019, 18, 717-724.

(16) Martínez, C.; Corma, A. Zeolites. Compr. Inorg. Chem. II 2013, $5,103-131$.

(17) Corma, A. Inorganic Solid Acids and Their Use in AcidCatalyzed Hydrocarbon Reactions. Chem. Rev. 1995, 95, 559.

(18) Brus, L. E. Electron-electron and electron-hole interactions in small semiconductor crystallites: The size dependence of the lowest excited electronic state. J. Chem. Phys. 1984, 80, 4403.

(19) Frisch, M. J., Trucks, G. W., Schlegel, H. B., Scuseria, G. E., et al. Gaussian 16, Revision C.01; Gaussian, Inc., 2009.

(20) Kamat, P. V.; Pradhan, N.; Schanze, K.; et al. Challenges and Opportunities in Designing Perovskite Nanocrystal Heterostructures. ACS Energy Lett. 2020, 5, 2253-2255.

(21) Ye, S.; Sun, J.-Y.; Han, Y.-H.; et al. Confining Mn2+-Doped Lead Halide Perovskite in Zeolite-Y as Ultrastable Orange-Red Phosphor Composites for White Light-Emitting Diodes. ACS Appl. Mater. Interfaces 2018, 10, 24656-24664.

(22) Sun, J.-Y.; Rabouw, F. T.; Yang, X.-F.; et al. Facile Two-Step Synthesis of All-Inorganic Perovskite $\mathrm{CsPbX} 3(\mathrm{X}=\mathrm{Cl}, \mathrm{Br}$, and I) Zeolite-Y Composite Phosphors for Potential Backlight Display Application. Adv. Funct. Mater. 2017, 27, 1704371.

(23) Akkerman, Q. A.; Park, S.; Radicchi, E.; et al. Nearly Monodisperse Insulator Cs4PbX6 (X $=\mathrm{Cl}, \mathrm{Br}, \mathrm{I})$ Nanocrystals, Their Mixed Halide Compositions, and Their Transformation into CsPbX3 Nanocrystals. Nano Lett. 2017, 17, 1924-1930.

(24) Wang, P.; Wang, B.; Liu, Y.; et al. Ultrastable Perovskite-Zeolite Composite Enabled by Encapsulation and In Situ Passivation. Angew. Chem., Int. Ed. 2020, 59, 23100-23106.

(25) Qian, J.; Guo, Q.; Liu, L.; et al. A theoretical study of hybrid lead iodide perovskite homologous semiconductors with $0 \mathrm{D}, 1 \mathrm{D}, 2 \mathrm{D}$ and 3D structures. J. Mater. Chem. A 2017, 5, 16786.

(26) Yin, J.; Maity, P.; De Bastiani, M.; et al. Molecular behavior of zero-dimensional perovskites. Sci. Adv. 2017, 3, No. e1701793.

(27) Kong, Z.-C.; Liao, J.-F.; Dong, Y.-J.; et al. Core@Shell CsPbBr3@Zeolitic Imidazolate Framework Nanocomposite for Efficient Photocatalytic CO2 Reduction. ACS Energy Lett. 2018, 3, $2656-2662$. 